ORIGINAL ARTICLE

\title{
Infant feeding and components of the metabolic syndrome: findings from the European Youth Heart Study
}

\author{
D A Lawlor, C J Riddoch, A S Page, L B Andersen, N Wedderkopp, M Harro, D Stansbie, \\ G Davey Smith
}

Arch Dis Child 2005;90:582-588. doi: 10.1136/adc.2004.055335

See end of article for authors' affiliations

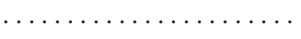

Correspondence to: Dr D A Lawlor, Department of Social Medicine University of Bristol, Canynge Hall, Whiteladies Rd, Bristol BS8 2PR, UK; d.a.lawlor@bristol.ac.uk

Accepted 15 October 2004

\begin{abstract}
Aims: To assess the associations of type and duration of infant feeding with components of the metabolic syndrome in children aged 9 and 15 .

Methods: A total of 2192 randomly selected schoolchildren aged 9 and 15 years from Estonia $(n=1174)$ and Denmark $(n=1018)$ were studied. Insulin resistance (homoeostasis model assessment), triglyceride levels, high density lipoprotein cholesterol, and systolic blood pressure were measured.

Results: Children who had ever been exclusively breast fed had lower systolic blood pressures than those who were not. With full adjustment for age, sex, country, birth weight, pubertal stage, body mass index, height, maternal and paternal education, income, smoking, and body mass index the mean systolic blood pressure of children who had ever been breast fed was $1.7 \mathrm{~mm} \mathrm{Hg}(95 \% \mathrm{Cl}-3.0$ to -0.5$)$ lower than those who had never been exclusively breast fed. There was a dose-response in this association with decreasing mean systolic blood pressure across categories from never exclusively breast fed to breast fed for more than six months. Exclusive breast feeding was not associated with other components of the metabolic syndrome. Results were similar when examined separately in each country.

Conclusions: The magnitude of the association, its independence of important confounding factors, and the dose-response suggest that exclusive breast feeding is causally associated with reduced systolic blood pressure. The magnitude of the effect we found with blood pressure is comparable to the published effects of salt restriction and physical activity on blood pressure in adult populations, suggesting that it is of public health importance.
\end{abstract}

$\mathrm{T}$ here is conflicting evidence concerning the role of breast feeding in infancy with later development of cardiovascular disease, with some, ${ }^{1-3}$ though not all, ${ }^{45}$ reporting a beneficial effect. Many more studies have assessed the association between breast feeding and subsequent blood pressure at different ages. A recent meta-analysis of these studies found a modest beneficial effect of breast feeding, which the authors concluded was unlikely to have important public health effects. ${ }^{6}$ The reviewers were unable to determine whether study participants had been exclusively breast fed for a number of studies, and for others mixed and exclusively breast fed children were compared to those who were not breast fed. Thus any beneficial effect of exclusive feeding may have been diluted in their overall pooled estimate. In addition, different confounders were taken into account in different studies and several studies were unable to adjust for important confounders in particular socioeconomic factors. ${ }^{7}$

Few studies examining the association between breast feeding and blood pressure have assessed a dose-response, which is important in determining causality. One large study, published since the review and meta-analysis, found that at age 7.5 years children who had ever been breast fed had mean systolic blood pressures that were on average $0.8 \mathrm{~mm} \mathrm{Hg}$ lower than for those who had never been breast fed. ${ }^{8}$ Further, in that study a dose-response, with greater duration of breast feeding being associated with greater reductions in blood pressure, was found. ${ }^{8}$ The authors concluded that the observed effects, if causal, would have important public health implications. Similar results in other populations would be supportive of a causal association.

Breast feeding has also been found to be associated with lower total cholesterol in adulthood ${ }^{9}$ and with reduced obesity in later life. ${ }^{10}$ High blood pressure, dyslipidaemia, and obesity are part of a complex of cardiovascular disease risk factors, which cluster in individuals, including in childhood, and are associated with increased cardiovascular disease-the insulin resistance or metabolic syndrome. ${ }^{11}$ Insulin resistance is associated with other early life risk factors including low birth weight and poor socioeconomic position, ${ }^{12}{ }^{13}$ and may be an important mediating factor between early life adverse exposures and later cardiovascular disease risk. ${ }^{14}{ }^{15}$ Although it has been suggested that breast feeding may protect against the development of type 1 diabetes in childhood in some, ${ }^{16}{ }^{17}$ though not all studies, ${ }^{18}$ the association between breast feeding and insulin resistance in a general population of children has not been previously assessed.

The aim of this study is to assess the association of being exclusively breast fed with insulin resistance and other components of the metabolic syndrome in children from two diverse populations-in Estonia and Denmark.

\section{METHODS}

Setting

Data from the European Youth Heart Study (EYHS) were used. The EYHS is a multicentre, international study addressing the prevalence and aetiology of cardiovascular disease risk factors in children aged 9 and 15 years. ${ }^{19}$ In the main study children from Estonia, Denmark, Norway, and Portugal were examined and completed health questionnaires. For the purposes of this paper, relevant data are available from children from two defined populationsEstonia (city and county of Tartu) and Denmark (city of Odense). Tartu is the second city of Estonia-an emerging eastern European country and former member of the Soviet Union. Odense is the third city of Denmark and is situated on the island of Fyn. We therefore have data on children from two very different physical and cultural environments representing eastern and northern Europe. Similar protocols 
for data collection were used in each country. Both study protocols conformed to the international guidelines on biomedical research and each research team complied with the ethical procedures of that country. Written, informed consent was obtained from the child's parent or legal guardian after they were given, in writing a full explanation of the aims of the study, its possible hazards, discomfort, and inconvenience. In addition, children had all the procedures verbally explained to them, together with any possible discomfort they might encounter and were given the option to withdraw at any time.

\section{Participants}

Boys and girls aged 9 and 15 years old were randomly selected to participate. These age groups were chosen to broadly represent children either side of puberty. At each study location, a defined population of children was identified and from this population a two stage cluster sample of children was randomly selected. The primary sampling units were schools, and secondary units were school registers. The overall participation proportion was similar in each country (76\% in Estonia and 75\% in Denmark). Fear of having a venous blood sample was the most frequently cited reason for non-participation by the children; in total, 2194 children participated.

\section{Measurements}

Each child underwent a physical examination including measurement of weight, height, waist and hip circumference, and blood pressure using standard procedures. All equipment used for blood pressure and anthropometric measures was the same in the two countries. Measurements were conducted in Denmark between September 1997 and June 1998 and in Estonia between September 1998 and June1999. The same protocols were used in both countries. Blood pressure was measured using either a Dinamap paediatric or adult vital signs monitor (model XL) with the choice between paediatric or adult machine and cuff size based on the child's arm circumference. Five measurements were taken at two minute intervals with the mean of the final three measurements used in all analyses. Blood samples were taken after an overnight fast and were all analysed at one central laboratory in Bristol, UK. High density lipoprotein cholesterol (HDLc), triglycerides, and glucose were measured by colorimetry using a Hitachi random access analyser. Insulin was measured by immunoassay, using a Dako assay that does not importantly cross-react with pro-insulin. Insulin resistance was estimated according to the homoeostasis model assessment (HOMA) as the product of fasting glucose $(\mathrm{mmol} / \mathrm{l})$ and insulin $(\mu \mathrm{U} / \mathrm{ml})$ divided by the constant $22.5 .^{20}$

Waist circumference was measured, at the end of gentle expiration, with a metal anthropometric tape midway between the lower rib margin and the iliac crest. The measurement was taken at right angles to the axial line of the trunk. Hip circumference was measured over the buttocks, normally at the level of the great trochanter but not lower than symphysis level. Both measurements were taken at right angles to the axial line of the trunk. Weight was measured in light clothing to the nearest $0.1 \mathrm{~kg}$ using a calibrated beam balance scale. Height was measured with-

out shoes to the nearest $0.5 \mathrm{~cm}$ using a transportable Harpenden stadiometer. The beam balance was calibrated using a weight of known mass and the stadiometer was calibrated with a metal measuring tape. Body mass index (BMI) was calculated as weight $(\mathrm{kg}) /$ height $(\mathrm{m})^{2}$. Pubertal status was assessed by trained personnel using Tanner's classification. ${ }^{21}$

Both of the children's parents at the time of their examination were asked to complete a questionnaire. Mothers were asked to respond to two questions concerning breast feeding:

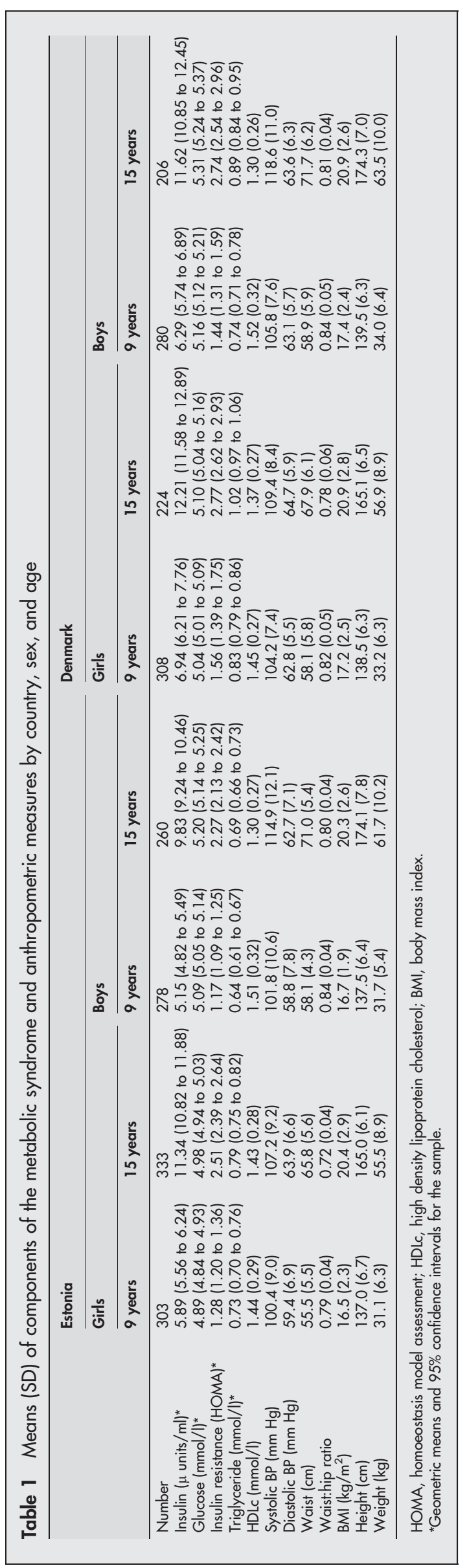


- Was your child fed completely on breast milk for any length of time-that is, without complementary bottle feeds?

- If yes, for how long was your child breast fed? (categories provided for response were: $<1 ; 1-3 ; 4-6$; $>6$ months)

In this paper we have compared children who were ever exclusively breast fed (answered yes to the first question) to those whose mothers indicated that they were never exclusively breast fed. A dose-response effect was examined by assessing levels of outcomes across the categories: never, $<1,1-3,4-6,>6$ months of exclusive breast feeding.

In the questionnaires both mothers and fathers were asked to report their weight and height, smoking status, educational attainment, and personal income.

\section{Statistical analysis}

Linear regression was used to estimate age, sex, and pubertal stage adjusted differences in insulin resistance and other characteristics between the two countries. Linear regression was used to assess the association between breast feeding and insulin resistance and other components of the metabolic syndrome with adjustment for potential confounding and mediating factors. F-tests were used to assess evidence of statistical interactions-between breast feeding and sex, age group, and country in these associations. Insulin, glucose, HOMA scores, and triglyceride levels were positively skewed; geometric means are shown and the natural log of the values were used in the regression models. In analyses in which HOMA scores and triglyceride levels were the outcome of interest, the resultant regression coefficients were back transformed to give a ratio of geometric means from which a proportional $(\%)$ difference by infant feeding was calculated. Robust standard errors, taking into account possible non-independence between children from the same country, were used to calculate $\mathrm{p}$ values and confidence intervals for analyses combining data from both countries. All analyses were conducted using Stata version 8.0 (Stata Corporation, Texas, 2002).

\section{RESULTS}

Table 1 shows the distributions of insulin resistance and the components of the metabolic syndrome and anthropometric measurements by country, sex, and age group of the children. As one would expect from previous studies, in both countries and among both girls and boys, the means of most risk factors were greater among the older than younger age groups and HDLc was lower in older age groups. In both countries and both age groups girls tended to have higher insulin levels and to be more insulin resistant than boys, and also had higher triglyceride levels. HDLc levels were lower among girls than boys at age 9, but were higher among girls than boys at age 15. All measures of obesity and components of the metabolic syndrome tended to be worse for children in Denmark than in Estonia (tables 1 and 2). The one exception was HDLc levels, which did not differ between the countries. Danish children were taller than Estonian children. There was slight attenuation of the country differences in components of the metabolic syndrome with adjustment for body mass index and waist to hip ratio, but essentially these differences remained even with adjustment for body mass index, waist to hip ratio, and height. For example, HOMA scores were 14\% (95\% CI 8 to 19) higher in Danish compared to Estonian children with adjustment for sex, age, pubertal stage, body mass index, waist to hip ratio, and height. Similar differences in triglyceride levels, systolic blood pressure, and diastolic blood pressure were $19 \%(95 \%$ CI 16 to 23$)$, $2.6 \mathrm{~mm} \mathrm{Hg}$ (95\% CI 1.9 to 3.4 ), and $2.2 \mathrm{~mm} \mathrm{Hg}$ (95\% CI 1.7 to 2.8 ) respectively.

Of the 1174 participants from Estonia, 1073 (91\%) of the mothers provided data on breast feeding, and of the 1020 Danish participants, 997 (98\%) of the mothers provided these data. There were no differences in insulin resistance or other components of the metabolic syndrome between those with breast feeding data and those without these data (all $\mathrm{p}$ values $>0.5$ ).

\section{Infant feeding and potential confounding factors}

Table 3 shows the distributions of potential confounding factors by whether the child was ever or never exclusively breast fed. Breast feeding rates were similar in both countries, with over $80 \%$ of children in both countries having been breast fed exclusively for some time in their lives. Children from the younger aged cohort (9 years) were more likely to have been breast fed than those from the older aged cohort (15 years). Those whose mothers had completed higher education were more likely to be breast fed, and there was a tendency for those whose mothers and fathers had higher personal income to be more likely to have been breast fed, though these associations were not statistically significant. Children with a higher birth weight were more likely to have been breast fed: mean difference $0.10 \mathrm{~kg}$ (95\% CI 0.03 to $0.16 ; p=0.005)$. Maternal and paternal body mass index were similar in those who had ever or never been breast fed (both $\mathrm{p}$ values $\geqslant 0.2$ ).

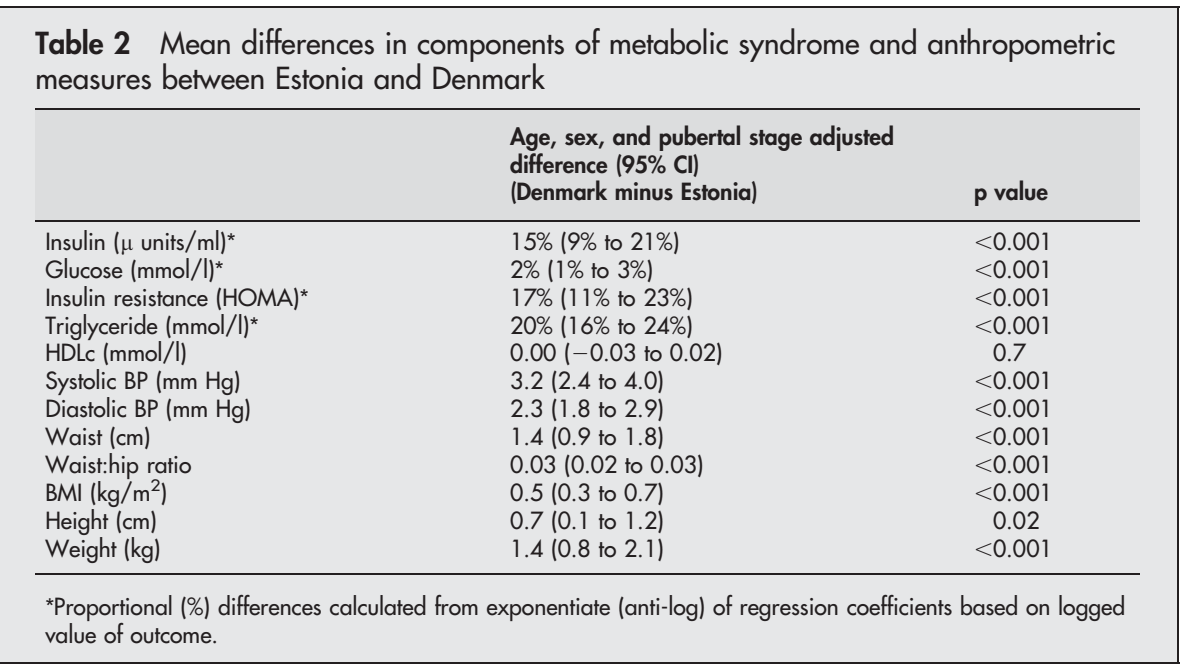




\begin{tabular}{|c|c|c|c|}
\hline Potential confounder & Number & $\%$ ever breast fed & p value* \\
\hline \multicolumn{4}{|l|}{ Country } \\
\hline Estonia & 1073 & 82.2 & \multirow[t]{2}{*}{0.2} \\
\hline Denmark & 997 & 84.6 & \\
\hline \multicolumn{4}{|l|}{ Sex } \\
\hline Female & 1107 & 82.7 & \multirow[t]{2}{*}{0.4} \\
\hline Male & 963 & 84.0 & \\
\hline \multicolumn{4}{|l|}{ Age group } \\
\hline 9 & 1127 & 85.7 & \multirow[t]{3}{*}{0.001} \\
\hline 15 & 943 & 80.3 & \\
\hline \multicolumn{3}{|c|}{$\begin{array}{l}\text { Pubertal stage (lower category indicates younger } \\
\text { biological age) }\end{array}$} & \\
\hline 1 & 915 & 86.0 & \multirow[t]{3}{*}{0.01} \\
\hline 2 & 715 & 80.6 & \\
\hline 3 & 425 & 82.4 & \\
\hline \multicolumn{4}{|l|}{ Maternal personal income } \\
\hline Low & 746 & 83.1 & \multirow[t]{3}{*}{0.5} \\
\hline Medium & 543 & 83.8 & \\
\hline High & 687 & 85.4 & \\
\hline \multicolumn{4}{|l|}{ Paternal personal income } \\
\hline Low & 607 & 82.7 & \multirow[t]{3}{*}{0.4} \\
\hline Medium & 456 & 84.2 & \\
\hline High & 672 & 85.6 & \\
\hline \multicolumn{4}{|l|}{ Maternal education } \\
\hline Compulsory only & 877 & 82.0 & \multirow[t]{3}{*}{0.05} \\
\hline Completed secondary & 736 & 83.6 & \\
\hline Higher or further & 371 & 86.4 & \\
\hline \multicolumn{4}{|l|}{ Paternal education } \\
\hline Compulsory only & 900 & 82.6 & \multirow[t]{3}{*}{0.09} \\
\hline Completed secondary & 669 & 83.9 & \\
\hline Higher or further & 316 & 86.4 & \\
\hline \multicolumn{4}{|c|}{ Maternal smoking at time of child's examination } \\
\hline Never & 940 & 84.6 & \multirow[t]{3}{*}{0.2} \\
\hline Past & 424 & 84.2 & \\
\hline Current & 682 & 81.7 & \\
\hline \multicolumn{4}{|c|}{ Paternal smoking at time of child's examination } \\
\hline Never & 576 & 84.6 & \multirow[t]{3}{*}{0.7} \\
\hline Past & 380 & 84.2 & \\
\hline Current & 952 & 83.1 & \\
\hline
\end{tabular}

\section{Infant feeding and components of the metabolic syndrome}

Examination of stratum specific estimates and F-tests suggested that there were no interactions with sex, age, or country in any of the associations between infant feeding and insulin resistance or components of the metabolic syndrome (all $\mathrm{p}$ values $>0.4$ ). The remaining results from multivariable analyses are therefore presented largely with all participants combined. These multivariable analyses were performed only on participants with complete data on all variables included in any of the models, so that comparisons between estimates from different models are based on the same subgroups. Of the 2070 participants with breast feeding data, 1557 (75\%) had complete data on all other variables included in the models. There were no differences in HOMA scores or other components of the metabolic syndrome between those with these complete data and those without (all p values $>0.2$ ).

Children who had ever been exclusively breast fed had lower systolic blood pressures than those who had never been exclusively breast fed: mean difference with adjustment for age, sex, country, and pubertal stage $-1.7 \mathrm{~mm} \mathrm{Hg}$ (95\% CI -3.0 to -0.4$)$. This association was slightly attenuated with adjustment for maternal body mass index to $-1.6 \mathrm{~mm} \mathrm{Hg}$ $(95 \%$ CI -2.9 to -0.3$)$ and increased slightly with adjustment for the child's own height: $-1.8 \mathrm{~mm} \mathrm{Hg}$ (95\% CI -3.1 to -0.5$)$. Other potential confounding factors had little effect on the association. With full adjustment for age, sex, country, birth weight, pubertal stage, body mass index, height, maternal and paternal education, income, smoking, and body mass index the mean systolic blood pressure of children who had been breast fed was $1.7 \mathrm{~mm} \mathrm{Hg}$ (95\% CI -3.0 to -0.5) lower than those who had never been exclusively breast fed. In stratified analyses the fully (for age, gender, birth weight, pubertal stage, body mass index, height, maternal and paternal education, income, smoking, and body mass index) adjusted difference between ever and never being exclusively breast fed in Estonia was $-1.7 \mathrm{~mm} \mathrm{Hg}$ (95\% CI -3.7 to 0.3 ) and in Denmark was $-2.0 \mathrm{~mm} \mathrm{Hg} \mathrm{(95 \%}$ CI -3.6 to -0.5$)(p=0.8$ for difference between country effects). Similar stratified analyses by gender produced fully adjusted differences of $-1.9 \mathrm{~mm} \mathrm{Hg}(95 \% \mathrm{CI}-3.4$ to -0.5$)$ among girls and $-2.0 \mathrm{~mm} \mathrm{Hg}(95 \% \mathrm{CI}-4.1$ to 0.0$)$ among boys, and by age group the fully adjusted differences were $-1.4 \mathrm{~mm} \mathrm{Hg}(95 \% \mathrm{CI}-3.3$ to 0.5$)$ among 9 year olds and $-2.4 \mathrm{~mm} \mathrm{Hg}$ (95\% CI -4.0 to -0.8 ) among 15 year olds.

There were no associations between exclusive breast feeding and insulin resistance, triglyceride levels, HDLc, or waist to hip ratio either when all data were combined or when analyses were stratified by country, gender, or age group. For example, the sex, age, country, and pubertal stage adjusted differences between those who were ever, compared to never, breast fed were $-4 \%$ ( $95 \% \mathrm{CI}-13$ to 5 ) for HOMA score, $-1 \%$ (95\% CI -7 to 4 ) for triglycerides, $-0.02 \mathrm{mmol} / \mathrm{l}$ (95\% CI -0.06 to 0.02 ) for HDL, and 0.00 (95\% CI -0.01 to 0.01 ) for waist to hip ratio. Although not conventionally considered part of the metabolic syndrome, we also examined associations between breast feeding and total and LDL cholesterol and found no associations with either of these outcomes. The sex, age, country, and pubertal stage adjusted differences between those who were ever, compared to 


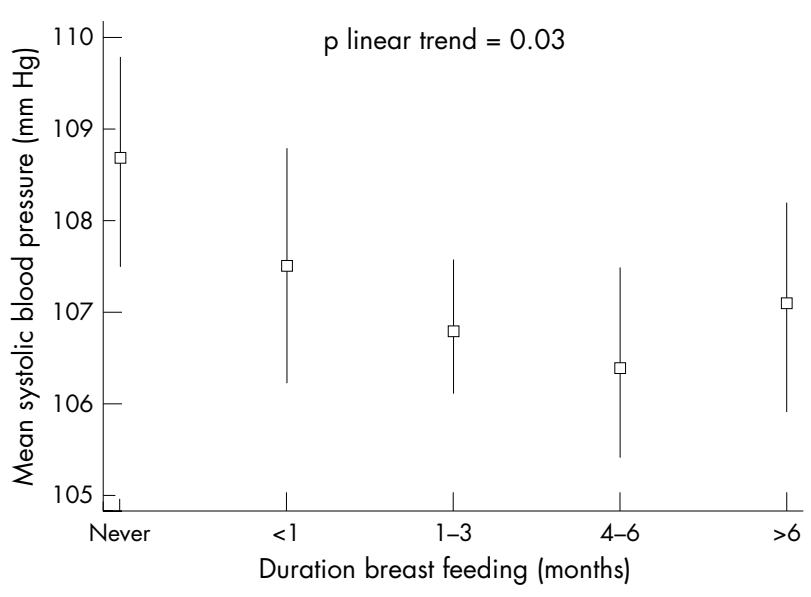

Figure 1 Adjusted mean systolic blood pressure by duration of exclusive breast feeding (adjusted for: child's age, sex, country, pubertal stage, BMI, height; mother's education, income, smoking, BMl; father's education, income, smoking, BMII).

never, breast fed were $-0.02 \mathrm{mmol} / \mathrm{l}(95 \% \mathrm{CI}-0.11$ to 0.07$)$ for total cholesterol and $0.04 \mathrm{mmol} / \mathrm{l} \quad(95 \%$ CI -0.08 to 0.15 ) for LDLC.

There was a dose-response between exclusive breast feeding duration and mean systolic blood pressure (table 4 , fig 1), with decreasing blood pressure across categories from never to at least six months of exclusive breast feeding. Other components of the metabolic syndrome did not show a dose response. The dose-response did not differ by country $(\mathrm{p}=0.4)$.

\section{DISCUSSION}

Among children aged 9 and 15 years from Estonia and Denmark we have found that exclusive breast feeding is associated with lower systolic blood pressure, and that there was a dose-response between breast feeding duration and systolic blood pressure. This association was independent of a number of potential confounding factors and was the same in both countries, despite major differences in sociocultural, dietary, and metabolic risk factors distributions between the countries. Breast feeding was not associated with other components of the metabolic syndrome.

\section{Study strengths and limitations}

The main strengths of this study are the availability of measures of insulin resistance and other components of the metabolic syndrome on these children together with maternal questions specifically aimed at establishing whether children were exclusively breast fed and for how long. In addition we had details of potential confounding factors including parental socioeconomic and smoking characteristics. The overall response proportion was good at $75 \%$, but of these participants only $75 \%$ had complete data on all variables included in multivariable models. Those with missing data did not differ from those without these missing data on the main outcomes of interest. Selection bias due to non-response or lack of data would only occur if associations among non-responders or those without complete data were either non-existent or in the opposite direction to those we have found, which seems unlikely.

We used retrospective maternal report of breast feeding behaviour. Our rates of breast feeding among the 9 year old children from Denmark were similar to those in one study from that country which collected data in 1994-95 at the time that mothers were breast feeding. ${ }^{22}$ However, we do not have similar information for Estonia, and for both countries routine statistics on breast feeding are not available in UNICEF documentation (http://www.unicef.org/nutrition/ index_statistics.html), suggesting that nationally representative data for these countries has not be routinely collected. Since mothers will not know the blood pressure or other metabolic outcomes of their children we can see no reason why the self-report of breast feeding will be differentiated by our outcome measures. Any non-differential measurement error would tend to dilute the associations we have presented. We were unable to obtain valid information on the content of formula feeds for the two countries over the relevant time periods. Given the differences between the two countries it is possible that formula content would differ between them, in which case the similarity of findings between the two countries would suggest that the mechanism for the association between breast feeding and systolic blood pressure is to do with breast feeding rather than the content of formula feeds. However, without the appropriate information we can only speculate about this.

Our assessment of potential confounding factors such as socioeconomic status and parental smoking and body mass index was made at the time of examining the child, and we do not have information on these variables at the time that the child was born or in infancy. Had we been able to measure these characteristics around the time of birth they may have been more strongly associated with breast feeding than the measurements made when the children were aged 9 and 15. Ideally, measures both around the time of birth and around the time at which the child's blood pressure was assessed would be the best way to deal with their potential confounding effect. However, these characteristics tend to be highly correlated over an individual's life course, and birth weight will act as a proxy indicator of socioeconomic status around birth; therefore residual confounding due to early life social factors is unlikely to fully explain the association we have found.

Table 4 Dose-response between exclusive breast feeding duration and insulin resistance and components of the metabolic syndrome among children from both Estonia and Denmark $(n=1557)$ with complete data on all variables

\begin{tabular}{|c|c|c|c|c|c|}
\hline \multirow{2}{*}{$\begin{array}{l}\text { Duration of } \\
\text { exclusive breast } \\
\text { feeding }\end{array}$} & \multicolumn{5}{|c|}{ Fully adjusted* difference $(95 \% \mathrm{Cl})$} \\
\hline & HOMA score & Triglycerides & HDLc & Systolic blood pressure & Waist to hip ratio \\
\hline $\begin{array}{l}\text { Never } \\
<1 \text { month } \\
1-3 \text { months } \\
4-6 \text { months } \\
>6 \text { months } \\
p \text { trend }\end{array}$ & $\begin{array}{l}0 \\
-11 \%(-22 \text { to } 0) \\
-2 \%(-13 \text { to } 8) \\
-3 \%(-14 \text { to } 8) \\
-3 \%(-14 \text { to } 8) \\
0.9\end{array}$ & $\begin{array}{l}0 \\
-1 \%(-8 \text { to } 7) \\
-2 \%(-8 \text { to } 4) \\
-4 \%(-11 \text { to } 2) \\
0 \%(-6 \text { to } 7) \\
0.7\end{array}$ & $\begin{array}{l}0 \\
-0.04(-0.09 \text { to } 0.02) \\
-0.01(-0.05 \text { to } 0.04) \\
-0.01(-0.06 \text { to } 0.04) \\
-0.05(-0.10 \text { to } 0.00) \\
0.2\end{array}$ & $\begin{array}{l}0 \\
-1.12(-2.87 \text { to } 0.61) \\
-1.85(-3.24 \text { to }-0.45) \\
-2.13(-3.69 \text { to }-0.58) \\
-1.56(-3.15 \text { to } 0.05) \\
0.03\end{array}$ & $\begin{array}{l}0 \\
0.00(-0.10 \text { to } 0.01) \\
0.00(-0.01 \text { to } 0.01) \\
0.00(-0.01 \text { to } 0.0) \\
0.00(-0.0 \text { to } 0.0) \\
0.8\end{array}$ \\
\hline
\end{tabular}

*For child's age, sex, country, birth weight, pubertal stage, BMI, height; mother's education, income, smoking, body mass index; father's education, income, smoking, body mass index. 


\section{Comparisons with other studies and possible mechanisms}

A recent meta-analysis of previous studies examining the association between breast feeding and systolic blood pressure found a pooled estimate from all studies of $-1.10 \mathrm{~mm} \mathrm{Hg}$ (95\% CI -1.79 to -0.42 ), but with evidence of small study bias. ${ }^{6}$ Among studies with more than 1000 participants (as in our study) the pooled estimate was $-0.16 \mathrm{~mm} \mathrm{Hg}$ (95\% CI -0.60 to 0.28 ), and the authors concluded that selective publication of small studies with positive findings may have exaggerated claims that breast feeding reduces systolic blood pressure in later life. ${ }^{6}$ In that review only four of a total of 26 studies included more than 1000 participants. ${ }^{6}$ Of those four, three established exclusive breast feeding but each had different exposures: two exclusive breast feeding for the first three months compared to bottle feeding; one exclusive breast feeding for at least three months versus never; and the fourth exclusive or mixed feeding for at least three months versus no breast feeding. ${ }^{6}$ None presented data on a dose response. Our study and one other large study $(\mathrm{n}=4763)^{8}$ published since the review suggest that ever being exclusively breast fed is associated with a $1-2 \mathrm{~mm} \mathrm{Hg}$ reduction in systolic blood pressure in childhood and that there is a dose response in the association. The magnitude of the association we have found, its independence of a number of potential confounding factors, and the dose response, together with similar findings from another large study, ${ }^{8}$ support a causal association between breast feeding and reduced systolic blood pressure.

We are not aware of any previous study that has assessed the association between breast feeding and insulin resistance. A large number of studies have assessed this association with body mass index and other measures of adiposity in later life. ${ }^{10}$ The results from these studies are conflicting, but it appears that once socioeconomic factors and parental size are taken into account there is no strong evidence of an association between breast feeding and later adiposity. ${ }^{7}{ }^{10}$ The small number of studies that have examined the association between breast feeding and lipid outcomes have also reported conflicting results. ${ }^{9}$ However, a systematic review found that differences between studies was in part explained by varying effects with age at which the lipid assays were undertaken. ${ }^{9}$ Thus, in infancy breast feeding was associated with increased total and LDLc, whereas in adulthood it was associated with reduced levels of these outcomes. If this interaction with age is a real effect, then our study of individuals assessed in later childhood and adolescence may have found null results because this age represents a transition period between the opposing infancy and adult effects. However, further evidence from large prospective birth cohorts is required to be certain that the direction of the effect of breast feeding on lipid levels does indeed vary with age.

If there is truly a specific association with blood pressure, but not other components of the metabolic syndrome, then this suggests a mechanism involving intermediaries that specifically affect blood pressure and not insulin resistance or dyslipidaemia. Breast feeding may be associated with lower blood pressure through a hormonal effect or may be related to lower levels of sodium in breast milk or a protective effect of long chain polyunsaturated fatty acids. Among adults, salt reduction is associated with small reductions in blood pressure. ${ }^{23}$ However, in a Dutch study in which infants were randomised to a low or a normal sodium diet for the first six months of life, blood pressure was markedly lower after 15 years in those allocated to the low sodium diet, suggesting that sodium restriction in infancy may have even greater effects on later blood pressure than salt reductions in adulthood. ${ }^{24}$ Long chain polyunsaturated fatty acids are present in breast milk, but not in most formula feeds, and these may be important in normal health and development of the infant. ${ }^{25}$ A recent follow up of a randomised controlled trial found that blood pressure at age 6 was lower (mean difference of systolic blood pressure $-3.0 \mathrm{~mm} \mathrm{Hg} \mathrm{(95 \%} \mathrm{CI}$ -5.4 to -0.5$)$ and of diastolic blood pressure $-3.6 \mathrm{~mm} \mathrm{Hg}$ $(95 \%$ CI -6.5 to -0.6$)$ ) among formula fed children who had been randomised to a formula supplemented with a long chain polyunsaturated fatty acid than among those randomised to an unsaturated formula. ${ }^{26}$ The blood pressure among the supplemented group was similar to that among 6 year olds whose mothers had breast fed them.

\section{Implications}

The magnitude of the effect that we have found between breast feeding and blood pressure in later childhood is similar to the effect of salt restriction $(-1.3 \mathrm{~mm} \mathrm{Hg})$ and physical activity $(-0.7 \mathrm{~mm} \mathrm{Hg})$ on blood pressure reported in a systematic review of non-pharmacological interventions in adults. ${ }^{23}$ It is possible that the effect of breast feeding on systolic blood pressure may amplify with increasing age, ${ }^{27}$ and indeed we found that the association was of a greater magnitude in 15 year olds than 9 year olds $(-2.4$ versus $-1.4)$, though this difference was not statistically significant. If the association we have found is causal, breast feeding may have important public health benefits with respect to blood pressure reduction and hence reduced risk of future cardiovascular disease. Breast feeding has a number of other important benefits for mother and offspring. The likely beneficial effects on future blood pressure can be added to this list and used in addition to other benefits in the promotion of breast feeding.

\section{ACKNOWLEDGEMENTS}

The authors are very grateful to the participants and their families who gave their time to the study. We would also like to acknowledge all members of the European Youth Heart Study Group not listed as co-authors of this paper.

\section{Authors' affiliations}

D A Lawlor, G Davey Smith, Department of Social Medicine, University of Bristol, UK

C J Riddoch, London Institute for Sport and Exercise, Middlesex

University, UK

A S Page, Department of Exercise and Health Sciences, University of Bristol, UK

L B Andersen, Department of Sports Medicine, Norwegian University of Sport and Physical Education, Oslo, Norway

N Wedderkopp, Institute of Sport Science and Clinical Biomechanics, University of Southern Denmark, Odense, Denmark

M Harro, Department of Public Health, University of Tartu, Tartu, Estonia D Stansbie, Department of Biochemistry, University of Bristol, UK

Funding: This study was supported by the following grants: Denmark: Danish Heart Foundation, Danish Medical Research Council, Health Foundation, Danish Council for Sports Research, Foundation of 17-121981, Foundation in Memory of Asta Florida Bolding nee Andersen, and Faculty of Health Sciences, University of Southern Denmark; Estonia: Estonian Science Foundation grant nos. 3277 and 5209. DAL is funded by a UK Department of Health Career Scientist Award. The views expressed in this paper are those of the authors and not necessarily any funding body.

Competing interests: none declared

\section{REFERENCES}

1 Osborn GR. Stages in development of coronary disease observed from 1,500 young subjects. Relationship of hypotension and infant feeding to aetiology. Colloques Internationaux du Centre National de la Recherche Scientifique 1967;169:93-139.

2 Osmond C, Barker DJ, Winter PD, et al. Early growth and death from cardiovascular disease in women. BMJ 1993;307:1519-24.

3 Barker DJ, Winter PD, Osmond C, et al. Weight in infancy and death from ischaemic heart disease. Lancet 1989;2:577-80. 
4 Burr ML, Beasley WH, Fisher CB. Breast feeding, maternal smoking and early atheroma. Eur Heart $J$ 1984;5:588-91.

5 Wingard DL, Criqui MH, Edelstein SL, et al. Is breast-feeding in infancy associated with adult longevity? Am J Public Health 1994;84:1458-62.

6 Owen CG, Whincup PH, Gilg JA, et al. Effect of breast feeding in infancy on blood pressure in later life: systematic review and meta-analysis. BMJ 2003;327: $1189-95$

7 Wadsworth M, Marshall S, Hardy R, et al. Breast feeding and obesity: relation may be accounted for by social factors. BMJ 1999;319:1576.

8 Martin RM, Ness AR, Gunnell D, et al, for the ALSPAC Study Team. Does breast-feeding in infancy lower blood pressure in childhood? The Avon Longitudinal Study of Parents and Children (ALSPAC). Circulation 2004; 109:1259-66.

9 Owen CG, Whincup PH, Odoki K, et al. Infant feeding and blood cholesterol: a study in adolescents and a systematic review. Pediatrics 2002; 1 10:597-608

10 Parsons TJ, Power C, Logan S, et al. Childhood predictors of adult obesity: a systematic review. Int J Obesity 1999;23:S1-107.

11 Liese AD, Mayer-Davis EJ, Haffner SM. Development of the multiple metabolic syndrome: an epidemiologic perspective. Epidemiol Rev 1998;20:157-72.

12 McKeigue PM, Lithell HO, Leon DA. Glucose tolerance and resistance to insulin-stimulated glucose uptake in mean aged 70 years in relation to size at birth. Diabetologia 1998:41:1133-8.

13 Lawlor DA, Ebrahim S, Davey Smith G. Socioeconomic position in childhood and adulthood and insulin resistance: cross sectional survey using data from British women's heart and health study. BMJ 2002;325:805-7.

14 Hales CN, Barker DJ. The thrifty phenotype hypothesis. Br Med Bull 2001;60:5-20.

15 Hattersley AT, Tooke JE. The fetal insulin hypothesis: an alternative explanation of the association of low birthweight with diabetes and vascular disease. Lancet 1999;353:1789-92.
16 Samuelsson U, Johansson C, Ludvigsson J. Breast-feeding seems to play a marginal role in the prevention of insulin-dependent diabetes mellitus. Diabetes Res Clin Pract 1993;19:203-10.

17 Borch-Johnsen K, Joner G, Mandrup-Poulsen T, et al. Relation between breast-feeding and incidence rates of insulin-dependent diabetes mellitus. A hypothesis. Lancet 1984;2:1083-6.

18 Virtanen SM, Knip M. Nutritional risk predictors of beta cell autoimmunity and type 1 diabetes mellitus at young age. Am J Clin Nutr 2003;78:1053-67.

19 Riddoch CJ, Andersen LB, Wedderkopp N, et al. Physical activity levels and patterns of 9 and 15 year old European children. Med Sci Sports Exerc 2004;36:86-92.

20 Matthews DR, Hosker JP, Rudenski AS, et al. Homeostasis model assessment: insulin resistance and beta-cell function from fasting plasma glucose and insulin concentrations in man. Diabetologia 1985;28:412-19.

21 Tanner JM. Growth at adolescence. Oxford: Blackwell, 1962.

22 Michaelsen KF, Larsen PS, Thomsen BL, et al. Duration of breast feedingwhich factors are significant? [in Danish]. Ugeskr Laeger 1995; 157:2311-15.

23 Ebrahim S, Davey Smith G. Lowering blood pressure: a systematic review of sustained effects of non-pharmacological interventions. J Public Health Med 1998;20:441-8.

24 Geleijnse JM, Hofman A, Witteman JC, et al. Long-term effects of neonatal sodium restriction on blood pressure. Hypertension 1997;29:913-17.

25 Koletzko B, Agostoni C, Carlson SE, et al. Long chain polyunsaturated fatty acids (LC-PUFA) and perinatal development. Acta Paediatr 2001;90:460-4

26 Forsyth JS, Willatts P, Agostoni C, et al. Long chain polyunsaturated fatty acid supplementation in infant formula and blood pressure in later childhood: follow up of a randomised controlled trial. BMJ 2003;326:953-5.

27 Singhal A, Cole TJ, Lucas A. Early nutrition in preterm infants and later blood pressure: two cohorts after randomized trials. Lancet 2001;357:413-19.

\section{ARCHIVIST}

\section{Acute stress and asthma}

T

here is evidence that psychosocial stress may both induce asthma and make pre-existing asthma worse. A study in Glasgow (S Sandberg and colleagues. Lancet 2000;356:982-7)

showed that among children with asthma a severely stressful life event increased the risk of an acute exacerbation 3-6 weeks after the event. High levels of chronic stress both increased the risk of an exacerbation of asthma after acute stress and reduced the length of time between the acute stress and the exacerbation. The dynamic logistic regression analysis used in that report used 2-week time periods and could not detect any effect of acute stress on asthma exacerbations soon after the stress. Now a survival analysis technique (hierarchical Cox regression) has been used on data from the same study to show that there is an effect in the first 2 days after acute stress (S Sandberg and colleagues. Thorax 2004;59:1046-51).

This analysis was restricted to 60 of the 70 children in the study who did not have high chronic stress. They were aged $6-13$ years (mean 8.5 years) and attending a hospital asthma clinic. They were followed up for 18 months with daily symptom diaries and peak flow measurements, and regular assessment of life events using a standardised interview (the Psychological Assessment of Childhood Experiences (PACE)). The children experienced 361 exacerbations of asthma and 124 severely negative life events during the study. During the first 2 days after a severely negative life event the risk of an asthma attack was increased 4.7fold. At 3-7 days after the event the risk was not increased but between 5 weeks and 7 weeks after the event the risk was again increased (by $80 \%$ ).

Among children with asthma, but without high levels of chronic stress, acute psychosocial stress has both an early and a late effect in increasing the risk of exacerbations of asthma. The early effect occurs in the first day or two after the stress and the late effect at weeks 5 to 7. The physiological mechanisms involved remain to be elucidated. 